\title{
Automated Urinalysis Machines: Potential Impact on the Diagnosis of Bladder Cancer
}

\author{
Edward Messing* \\ University of Rochester Medical Center, Rochester, NY, USA
}

Hematuria, both macroscopic and microscopic, is the most common finding leading to a diagnosis of bladder cancer. The American Urological Association (AUA) defines microhematuria as having $>3$ red blood cells (RBCs) per high-powered field on a microscopic urinalysis [1]. This has traditionally been performed by spinning $10-15 \mathrm{ml}$ of freshly voided urine in a centrifuge for 5 minutes at 3000 revolutions per minute and suspending the sediment in $1 \mathrm{cc}$ of urine or saline, placing it on a microscope slide and examining several microscopic fields at 40x power [2]. While many factors may greatly influence the count of RBCs, including the size and speed of the centrifuge, the volume of urine spun down, and the amount of fluid the sediment is resuspended in, this has been an accepted definition of microhematuria. Since newly diagnosed bladder cancers detected because of microscopic hematuria are more frequently detected at pre-muscle invasive stages than those associated with macroscopic hematuria [3] $(p=0.03)$, it is important that a standard minimum number of RBCs be widely accepted to define microhematuria [1]. Thus, a recently published article by Hertz and coworkers [4] describing several commercially available automated urinalysis machines, and differences in their performance and calibration is very important to all urologists, and particularly those who diagnose and treat bladder cancer [4]. These machines, which are in wide use throughout

\footnotetext{
${ }^{*}$ Correspondence to: Edward M. Messing, MD, FACS, University of Rochester Medical Center, Rochester, NY, USA. Tel.: +1 585275 3345; E-mail: Messing@urmc.rochester.edu.
}

the United States and other countries, utilize FLOW cytometry and spot images obtained with a microscopic camera that are analyzed by recognition software to distinguish the particles seen. Technicians often review these images as well to check their validity and then a measurement of RBCs (and other entities in the urine) is formulated. These are reported as RBCs/HPF based on correlations made with traditional microscopy on a limited number of samples when the machines are calibrated.

These machines can process 50-200 samples per hour, far more than the 30 or so an hour that can be reviewed by traditional microscopy, explaining their growing popularity in the past 5 years. The authors go on to describe the validation standards which differ for each machine as well as their correlations to the traditional microscopic urinalysis. Moreover, the quantification is usually put into groups (e.g. 0-4 $\mathrm{RBC} / \mathrm{HPF}, 5-10 \mathrm{RBC} / \mathrm{HPF}$, etc.), further clouding the correlation with traditional microscopy.

My colleagues at the University of Rochester and I were unaware that these machines existed, let alone that they were in such widespread use, and had no idea which machine(s) were at our hospitals. Indeed, we found out that at the three major hospitals in our healthcare system, three different machines are used. Also, we do not know which machines are used by other hospitals, from where we often are sent patients, and what their calibration standards are.

The concern, of course, is that without knowing those standards and how they correlate with microscopic urinalysis, we may not even be asked to see patients with microhematuria by the traditional 
definition. While bladder cancer is almost never found incidentally at autopsy and so will eventually be diagnosed, [5] this may occur at more advanced stages, leading to greater morbidity and possibly mortality for bladder cancer patients [3].

The take-home message for all of us is to at least know which machines are in use at the institutions where we work and their related laboratories, and try to influence the lab directors to calibrate their machines to a low number of RBCs (0-2 or 3) as the "normal group". Urologists are already somewhat derelict in performing cystoscopy in patients with microhematuria and increased risk factors for harboring urologic malignancies [6], and one would not want to increase this problem by relying on a relaxed or uncertain definition of microhematuria.

\section{CONFLICT OF INTEREST}

The author has no conflict of interest to report.

\section{REFERENCES}

[1] Davis R, Jones JS, Barocas DA, et al. Diagnosis, evaluation and follow-up of asymptomatic microhematuria (AMH) in adults. Am Urol Assoc. 2012. https://www.auanet.org/ guidelines/asymptomatic-microhematuria-(2012-reviewedfor-currency-2016). Accessed March 3, 2019.

[2] Brendler C. Evaluation of the urologic patient: History, physical examination and urinalysis. In: Campbell's Urology. 10th Ed. 2012:84-98.

[3] Ramirez D, Gupta A, Canter D, et al. Microscopic haematuria at time of diagnosis is associated with lower disease stage in patients with newly diagnosed bladder cancer. BJU Int. 2016;117;783-6.

[4] Hertz A, Perez DS, Anderson MI. Automated urinalysis for evaluation of microscopic hematuria: Current options and revising the "Gold Standard". Urology Practice, accepted for publication, 2019.

[5] Messing EM, Young TB, Hunt VB, et al. Home screening for hematuria: Results of a multi-clinic study. J Urol. 1992;148:289-292.

[6] David SA, Patil D, Alemozaffar M, et al. Urologist use of cystoscopy for patients presenting with hematuria in the United States. Urology. 2017;100:20-26. 